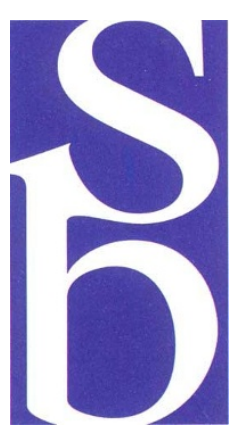

\section{Real and artificial histories}

Truth is stranger than fiction, especially when one considers the diversity of the biological world. Certainly one of the fascinations for life scientists is untangling the evolutionary threads that have lead to the world as we see it around us today. And this applies at every level of biology, from the structure of ecosystems to the structure of biological molecules. Two intriguing examples of the latter, that of the structure of a protein that has lost its catalytic function and taken on a purely structural role ${ }^{1}$ and of another enzyme that has a strikingly different mechanism of regulation in different organisms ${ }^{2}$, are provided in this issue of Nature Structural Biology.

\section{Eye of the turkey}

The crystallins are a motley collection of proteins - mainly enzymes - that have been 'hijacked' into performing an important structural role in the lens of the eye of all vertebrates (and a number of invertebrates, as well). Christine Slingsby and colleagues ${ }^{1}$ describe for the first time the structure of a $\delta$-crystalline from turkey lens. Unlike the $\alpha$-, $\beta$ - and $\gamma$-crystallins, which are ubiquitous in vertebrates, $\delta$-crystalline is known only in birds and reptiles. All the taxonspecific crystallins are closely related to enzymes: $\delta$-crystalline shows high sequence similarity and over $90 \%$ sequence identity - to argininosuccinate lyase (ASL), an enzyme involved in arginine biosynthesis and, in ureotelic species, the urea cycle.

Indeed, in some cases the crystallin and enzyme are one and the same protein produced from the same gene: in the duck it has been shown that $\varepsilon$-crystallin and lactate dehydrogenase- $\mathrm{B}$, and $\tau$-crystallin and $\alpha$-enolase are encoded by the same gene. This phenomenon of 'gene sharing' - where a protein has acquired a second, new function - is thought to have arisen through modification of gene expression. It is by such means that the ASL gene may have given rise to $\delta$-crystallin. In the chicken both genes have a lens-preferred enhancer suggesting that the ASL enzyme was recruited to the eye before gene duplication took place. Presumably the constraints of the new lens-specific function of ASL were not consistent with optimal enzyme function; these two independent sets of evolutionary pressures favouring gene duplication and further independent selection. It would seem that for all the crystallins the initial hijacking to the eye lens is essentially a neutral process - although most of the enzymes are oxo-reductases that bind pyridine nucleotide co-factors and these may confer advantage by acting as ultraviolet filters. Since then the gene for the lens-specific protein has accumulated mutations which compromise its now ancient enzymic function. Nonetheless, the new structure provides insight into the active site and catalytic mechanism of ASL ${ }^{1}$.

\section{Phosphorylating phosphorylase}

The allosteric mechanism by which rabbit muscle glycogen phosphorlyase is activated by phosphorylation of Ser 14 is - through the determination of the structure of both the active, phosphorylated enzyme and the inactive non-phosphorylated enzyme - well understood. That the structural elements involved in the allosteric control of the muscle enzyme are poorly conserved in yeast glycogen phosphorylase has suggested the mechanisms underlying phosphorylation-induced structural changes are different in yeast and mammals. This suspicion is borne out by the structure of the inactive, non-phosphorylated yeast enzyme, reported by Virginia Rath and Robert Fletterick ${ }^{2}$, and commented on in an associated News and Views article by Louise Johnson ${ }^{4}$.

Activation of the muscle enzyme by phosphorylation involves a number of linked events: a gate, consisting of a reverse turn, which blocks the entrance to the active site in the inactive muscle enzyme, opens; the homodimer subunit 
interface is drawn together; and the $\mathrm{N}$ - and Cterminal domains, which bound the active site within each subunit, separate. The inactive, non-phosphorylated yeast enzyme has features of the active muscle enzyme: the gate is already open; the catalytic residues are posed in positions equivalent to those in the activated muscle enzyme; and the subunits are drawn together. But the domains within the subunits have not separated to facilitate access to the active site. This seems to be in part because the $\mathrm{N}$ terminus, which is unique to the yeast enzyme, is stretched over the surface of the homodimer to the adjacent active site, and although it does not interfere directly with the catalytic residues it may prevent the approach of substrate and stabilize the'closed domain' orientation. Phosphorylation, which occurs 19 residues $\mathrm{C}$-terminal to the end of the extended arm, presumably alters the conformation of the arm, removing its inhibitory effects on the symmetry-related active site allowing activation.

For yeast and muscle glycogen phosphorylases, control by phosphorylation seems to have arisen independently during evolution; and both mechanism differ from that used by isocitate dehydrogenase. This situation can be contrasted to that in isocitrate dehydrogenase where the phosphate moiety plays a direct role in preventing the negatively charged citrate substrate from binding. So it would seem that for regulation by phosphorylation no particular mechanism need be adhered to, rather there is very likely as many answers - some more different than others, for sure - as there are enzymes regulated in this fashion.

\section{Test-tube evolution}

While these 'real' evolutionary histories tell us much about the construction of biomolecules our incomplete understanding of the biophysical parameters that determine structure and function mean that as yet we cannot fully appreciate either the constraints on - or the possibilities of - the different activities of RNA, DNA or proteins. Generating 'artificial' evolutionary histories does not require a full understanding of molecular architecture in order to create molecules with particular/unique functions, only that it is possible to generate a large population of molecules having a random distribution of structural elements and that there is an effective screening/selection procedure which allows identification of those molecules that have the desired characteristics.

RNA has been a particularly attractive candidate for such studies, given the relative ease with which large numbers of mutant molecules can assembled and assayed. The recent report of the evolution of a ribozyme that can function as a polynulclotide kinase $e^{5}$ builds on earlier work of selecting RNAs with the ability to bind ATP. Perhaps the most notable aspect of this study is the frequency with which reasonably active kinase ribozymes were recovered, given the vanishing small fraction of available sequence space sampled; $10^{16}$ out of a possible $10^{60}$ RNA molecules. Clearly, starting with a population of aptamers that can already bind the substrate greatly increases the chances of finding RNAs with the desired kinase activity. The use of catalytic antibodies to complement auxotrophic microorganisms provides an example of one way in which protein sequence space may be explored ${ }^{6}$. And protocols such as that for in vitro homologous recombination of pools of selected mutant genes - DNA shuffling ${ }^{7}$ - should provide a means of generating the necessary sequence diversity. Whatever the technology used, the ability to effectively mutagenise and select or screen proteins (or, indeed, any molecule of interest), on a large scale for defined characteristics will have profound implications for both basic research and medicine.

1. Simpson, A. et al. Nature Struct. Biol. 1, 724-734

2. Rath, V.L. \& Fletterick, R.J. Nature Struct. Biol. 1, 681-690

3. Piatigorsky, J. \& Wistow, G. Science 252, 1078-1079 (1991)

4. Johnson, L.N. Nature Struct. Biol. 1, 657-659

5. Lorsch, J.R. \& Szostak, J.W. Nature 371, 31-36 (1994)

6. Tang, Y., Hicks, J.B. \& Hilvert, D. Proc. natn. Acad.Sci U.S.A 88, 8784-8786 (1991)

7. Stemmer, W.P.C Nature 370,389-391 (1994) 